\title{
Wideband Wireless Access Systems Interference Robustness: Its Effect on Quality of Video Streaming
}

\author{
${ }^{1}$ Aderemi A. Atayero, ${ }^{2}$ Oleg I. Sheluhin \\ ${ }^{1,4}$ Department of Electrical and Information Engineering \\ Covenant University \\ Ota, Nigeria
}

\begin{abstract}
A necessary requirement incumbent on any information communication system and/or network is the capacity to transmit information with a predefined degree of accuracy in the presence of inevitable interference. The transmission of audio and video streaming services over different conduits (wireless access systems, Internet, etc.) is becoming ever more popular. As should be expected, this widespread increase is accompanied by the attendant new and difficult task of maintaining the quality of service of streaming video. The use of very accurate coding techniques for transmissions over wireless networks alone cannot guarantee a complete eradication of distortions characteristic of the video signal. A softwarehardware composite system has been developed for investigating the effect of single bit error and bit packet errors in wideband wireless access systems on the quality of H.264/AVC standard bursty video streams. Numerical results of the modeling and analysis of the effect of interference robustness on quality of video streaming are presented and discussed.
\end{abstract}

Keywords-codec; H.264/AVC; polynomial approximation coding; signal-to-noise ratio; video streaming.

\section{INTRODUCTION}

One of the most important Quality of Service (QoS) parameters for wireless networks is the probability of occurrence of bit and packet errors measured by the Bit Error Rate (BER) and Packet Error Rates (PER) respectively. Neither single packet losses nor single bit errors can provide a comprehensive imitation modeling of fading channels. In digital systems, errors often occur in packets as a result of transmission conditions. Specifically, signals are attenuated during transmission and this consequently leads to grouping (packetization) of errors. A group of erroneous packets is essentially a sequence of packets that are either lost in transit or received with error after transmission over a communication channel within a given period of time. Burst Error Length (BEL) is defined as the number of erroneous packets included in a given group of errors.

\section{METHOD}

The H.264/AVC standard is a compendium of innovations and improvements on prior video coding technologies vis-à-vis enhancement of coding efficiency and effective usage over a wide gamut of networks and applications [1]. For a complete analysis of the impact of errors on resultant signal quality, we investigate the influence of the conduit's (i.e. wireless transmission medium's) robustness on the perceivable quality

\author{
${ }^{3}$ Yuri A. Ivanov, ${ }^{4}$ Julet O. Iruemi \\ ${ }^{2,3}$ Department of Information Security \\ Moscow Tech. Univ. of Communication and Informatics \\ Moscow, Russia
}

of streaming video standard H.264/AVC using the developed hardware and software complex (HSC) [2], [3].

Objective and subjective indicators of video quality were obtained using methods described in [3]. For qualitative assessment, it is imperative to have the video file data before transmission over the network (on the transmitting end), and after reception from the network (at the receiving end).

Data required for the qualitative assessment at the transmitting end are:

a)the original unencoded video in YUV format,

b)the encoded video in MPEG-4,

c)transmission start time and

d)type of each packet sent to the network.

The following data are required for qualitative assessment at the receive end:

a)time of reception of each packet from the network,

b)type of each packet received from the network,

c)the encoded video (possibly distorted) in MPEG-4 format, and

d) the decoded video in YUV format for display.

We performed data evaluation by comparing the transmitted and received files.

\section{Structure of the Hardware-Software Complex (HSC)}

Data processing is carried out in the three phases described below:

First phase: the time taken in sending and receiving each packet on both sides as well as the packet type are analyzed. This results in a record of the type of frame and the time elapsed between transmitted and received packets. The distorted video file at the receive end is restored using the originally encoded video file and information about lost packets. Subsequently, the video is decoded for playback to the viewer.

Assessment of video quality is done at this stage. Video quality indicators always require a comparison of the received (possibly distorted) video frame and the corresponding source frame. In the case of a total loss of frame in transit, the necessary frame synchronization before and after transmission over the network becomes impossible. 
Second phase: In this phase of data processing, the problem of quality assessment is resolved based on the analysis of information about frame loses. Substituting the last relayed frame for the lost frame restores frame synchronization. This methodology allows for subsequent frame-wise assessment of video quality.

Third phase: At this stage, the assessment of the quality of decoded video is achieved by means of both the restored and source video files.

Fig. 1 shows a block diagram of the HSC for assessing the quality of streaming video. The schematic diagram reflects the interaction between modules in the transmission of the digital video from a source through the network connection to the viewer. The HSC modules interact with the network by using traces containing all the necessary data listed above. Thus, for proper functioning, the HSC requires two traces, the source video and the decoder. The data network can be considered simply as a two-port black-box that introduces delay, packet loss, and possibly packet rearrangement. The network was simulated based on the aforementioned assumptions [4] in the NS2 environment. A detailed description of the functional modules of the HSC is given in [3].

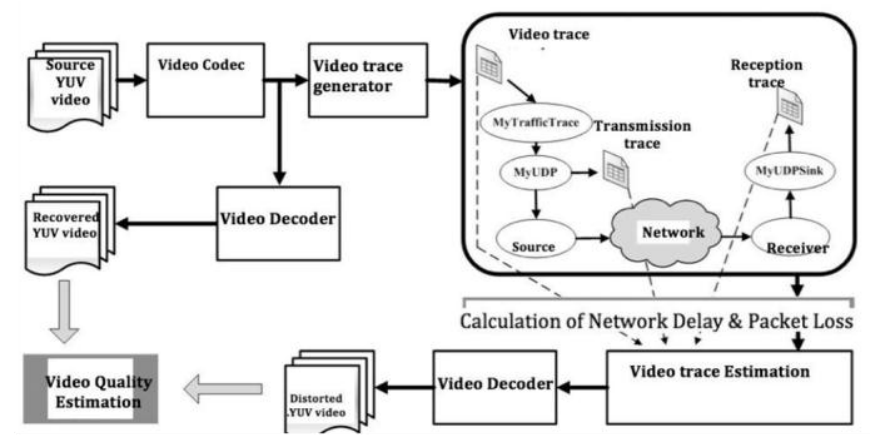

Figure 1. Block diagram of HSC.

\section{PRESENTATION OF DATA AND SiMUlation PREPARATION}

Video encoding begins with color space conversion from RGB to YUV also known as $\mathrm{Y}, \mathrm{Cr}, \mathrm{Cb}$ i.e. one luma and two chroma components [5]. It is common knowledge that there is a significant correlation of color components in any typical image of the RGB format, which makes it an obviously redundant format in terms of compression. The standard television uses a different representation of images, which also employs three components of the signal, but these components are uncorrelated (i.e. void of inter-componential redundancy). $\mathrm{R}, \mathrm{G}$ and $\mathrm{B}$ components are converted to luminance $\mathrm{Y}$ component and two color difference components $\mathrm{U}$ and $\mathrm{V}$ of the YUV format. Since most information is stored in the luminance component, little information is lost if a thinning of the $\mathrm{U}$ and $\mathrm{V}$ components is done.

Standard test videos in the YUV format may be used as initial test video sequences. However, these videos have limited playback time and hence do not allow for the assessment of change in quality under prolonged video broadcast. Similarly, a vast amount of experimental data cannot be obtained from them. It is for these reasons that we recorded our own 30- minute video in YUV format (send.YUV) with a resolution of $640 \times 480$ pixels and frame rate of 25fps using special software.

The first step is to encode the source video to H.264 format (video stream file). This is done by the video codec (a device used for encoding and decoding video signals). Video codecs are usually characterized by a) channel throughput, b) decoded video distortion rate, c) startup latency, d) end-to-end delay, computational complexity, and e) memory capacity. A good codec is one capable of providing the necessary trade-off vis-àvis these characteristics [5].

In the next step, the encoded video stream is packaged in an MP4-container for onward transport over the network using the User Datagram Protocol (UDP). The result of encoding the original video is an MP4-file. Since it is necessary to evaluate the quality of video transmitted over the network, the need arises to create a spare decoded YUV file from the newly created MPEG layer-4 file, which serves as the control in evaluating the quality of video transmitted over the network, excluding the impact of the codec. It is thus possible to estimate the influence of a wireless network on the received visual video quality, while excluding encoding and decoding losses.

For simulation purposes, it is necessary to create a video trace file that contains the following information: frame number, frame type, frame size, and the number of segments in which the frame is divided into packets. This video trace serves as the input to the simulator network, where the sending and reception of video data occurs. As a result of video transmission over the network, it is necessary to obtain transmission trace files and reception trace files, which contain the following packet data: the transmission/reception time, a unique identifier and trace file size. These two traces are used to determine lost packets in the network. In the end, we obtain files of the sent and received packets containing detailed information about the time of sending from the transmitter and the time of reception by the receiver.

\section{MOdEling AND Simulation OF TRANSMission OVER A WIRELESS NETWORK}

The HSC allows for the simulation of the main types of errors encountered when transmitting video data over wireless networks. The two types of simulation required are as listed below:

\section{A. Bit error simulation}

Simulated transmission over a wireless channel model with Additive White Gaussian Noise (AWGN) is conducted. In the process of simulation, certain bits in the sequence are distorted (i.e. inverted) with a given probability. The probability value used is defined by the Bit Error Rate (BER).

\section{B. Packet error simulation.}

The UDP packets can be manually deleted from the received trace file. This allows for the observation of codec functionality and analysis of change in visual quality in cases of packet loss. At the same time, both the received and undistorted files can be obtained during transmission over an "ideal" channel with unlimited bandwidth and no delay, with subsequent removal of some packets. 


\section{Calculation of Losses AND Estimation of}

TABLE 2. RELATIONSHIP BETWEEN QUALITY INDICATORS AND BER

\begin{tabular}{|c|c|c|c|c|}
\hline $\begin{array}{c}\text { PSNR } \\
{[\mathrm{dB}]}\end{array}$ & $\begin{array}{c}\mathrm{MOS} \\
{[\%]}\end{array}$ & BER & $\begin{array}{c}\text { ITU } \\
\text { Quality Scale }\end{array}$ & $\begin{array}{c}\text { Picture } \\
\text { Degradation }\end{array}$ \\
\hline$>37$ & $81-100$ & $<1 \times 10^{-4}$ & $\begin{array}{c}5 \\
\text { EXCELLENT }\end{array}$ & NOTICEABLE \\
\hline $31-37$ & $61-80$ & $1 \times 10^{-4}-4 \times 10^{-4}$ & $\begin{array}{c}4 \\
\text { GOOD }\end{array}$ & $\begin{array}{c}\text { NOTICEABLE, } \\
\text { BUT NOT } \\
\text { IRRITATING }\end{array}$ \\
\hline $25-31$ & $41-60$ & $4 \times 10^{-4}-8 \times 10^{-4}$ & $\begin{array}{c}3 \\
\text { SATISFACTORY }\end{array}$ & $\begin{array}{c}\text { SLIGHTLY } \\
\text { IRRITATING }\end{array}$ \\
\hline $20-35$ & $21-40$ & $8 \times 10^{-4}-1 \times 10^{-3}$ & $\begin{array}{c}2 \\
\text { POOR }\end{array}$ & IRRITATING \\
\hline$<20$ & $0-20$ & $\begin{array}{l}>1 \times 10^{-3} \\
\text { OBTAINED VID }\end{array}$ & $\begin{array}{l}\text { 1-VERY POOR } \\
\text { QUALITY }\end{array}$ & VERY IRRITATING \\
\hline
\end{tabular}

Calculation of losses given the availability of unique id of the package is quite easily achieved. With the aid of the video trace, each packet is assigned a type. Each package of the assigned type that is not included in the received trace is deemed lost. Loss of frame is calculated for any (and all) frame(s) with a lost packet. If the first packet in a frame is lost, then the whole frame is considered lost since the video decoder cannot decode a frame, which is missing the first part. Assessment of received traces is done by the module for trace assessment (see Fig. 1). The recovered file must be decoded in YUV format.

There are two major methods of estimating the quality of digital video, namely, the subjective and objective methods:

Subjective quality assessment is always based on viewer impression. It is extremely costly, very time consuming and requires specialized equipment. Traditionally, subjective video quality is determined by expert assessment and calculation of the average Mean opinion Score (MOS), which is assigned a value from 1 to 5 (ITU scale) [6], [7], where 1 and 5 represent worst and best received video quality respectively.

Objective video quality assessment is usually done by measuring the average luminance peak Signal-to-Noise Ratio (PSNR). The PSNR is a traditional metric, which allows for the comparison of any two images [8]. The PSNR module

TABLE 1. CHARACTERISTICS OF ENCODED VIDEO

\begin{tabular}{cc}
\hline \hline Format & MPEG-4 Part14 (MP4) \\
\hline Codec & H.264 \\
Bit rate & Constant @ 1150 kbps \\
Frame frequency & 25 fps \\
Resolution & $640 \times 480$ pixels \\
GOP type & IBBPBBPBB \\
\hline \hline
\end{tabular}

evaluates the objective quality of received video stream in polynomial approximation coding (PAC). The end result is the values of PSNR calculated for the original and distorted image (as shown in Fig. 2). MOS values are calculated from the PSNR indicator.

\section{ResUlTs, ANLYSIS AND DisCUSSION}

In order to study the effect of transmission errors on the resulting video quality, the transmission of a 30-minute video over a wireless network with random packet errors in the channel was simulated. Characteristics of sequences used are listed in Table I.

For modeling purposes, the encoded video stream was split into RTP/UDP-packets using the hardware-software tool reported in [3]. Bit error simulation for transmission over a wireless channel was done using an AWGN error generator contained within the PAC structure. Simulation of packet errors during transmission over a wireless channel was done by deleting packets from the received trace file [3]. This allowed us to explore and analyze the change in visual quality during loss of packets. The received and undistorted trace files were obtained for transmission over an "ideal" channel with unlimited bandwidth and no delay in using the NS2 software environment [4], followed by a random removal of packets, according to PER and BER parameters. Quality assessment was carried out using PSNR and MOS indicators, calculated by using hardware and software tools [4]. The standard deviation of the quality of the average PSNR values was calculated using equation (1) [9].

$$
S_{P S N R}^{\prime}=\sqrt{\frac{1}{N-1} \sum_{n=0}^{N-1}\left(P S N R_{n}-\overline{P S N R^{\prime}}\right)^{2}}
$$

\section{Effect of bit error}

Fig. 2 shows the effect of BER on the quality of video streaming.

Analysis of the results of streaming video over the simulated wireless network with different values of BER revealed the following:

i) Simulating a wireless channel using AWGN model, and additive, bit errors with a value of BER $\leq 3 \times 10^{-5}$ does not affect the quality of the video. However, when $\mathrm{BER} \geq 4 \times 10^{-3}$ packet loss in the network reaches its maximum value of $\geq 99,9 \%$.

ii) Objectively, excellent quality of video transmission over a channel can be guaranteed for all bit error probabilities less than $1 \times 10^{-4}$, good quality is in the range of $1 \times 10^{-4}$ to $4 \times 10^{-4}$, satisfactory quality is in the range of $4 \times 10^{-4}$ to $8 \times 10^{-4}$, poor quality is in the range of $8 \times 10^{-4}$ to $1 \times 10^{-3}$, while very bad quality is for any BER $>1 \times 10^{-3}$.

iii) The histograms of the distribution of PSNR values during simulation and broadcast over a real network in general are of a twin-peak form. One of the peaks characterizes the PSNR value of error-free video stream (the decoder is able to correct bit errors when they are relatively few in the frame). The second peak characterizes PSNR degradation due to the large number of corrupted video frames in fading moments (the decoder is unable to fix large numbers of bit errors). As the number of errors increases, this maximum increases commensurately with a decrease in the second. During transmission, depending on error 

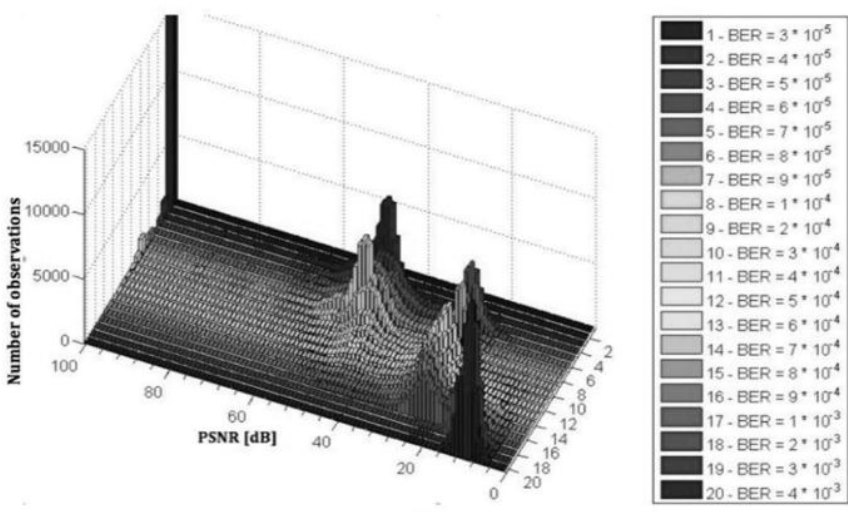

a)

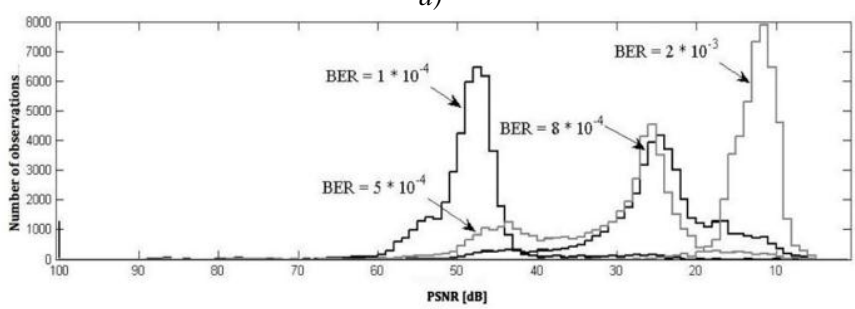

b)
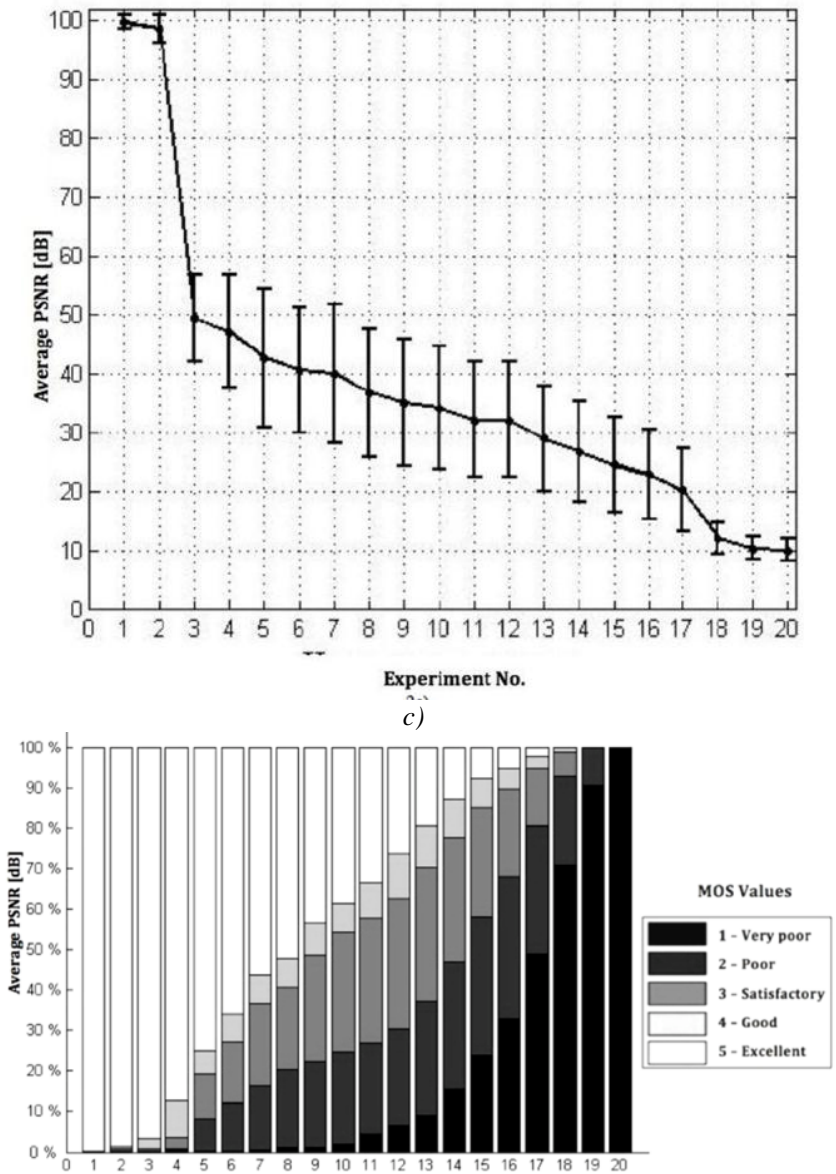

Experiment No.

d)

Figure 3. Values of video sequence quality indicators for different values of wireless channel BER: a) -PSNR value distribution histogram; b) - PSNR value distribution histogram for certain values of BER; c) - Quality deviation from average PSNR value; d) - quality gradation for MOS values.
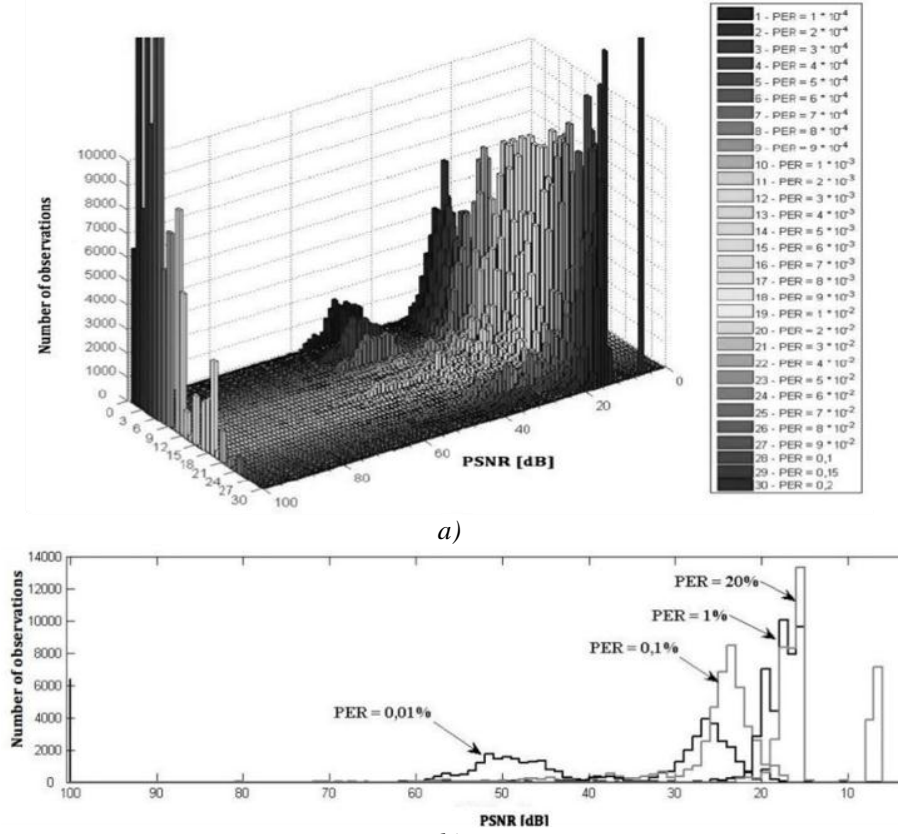

b)

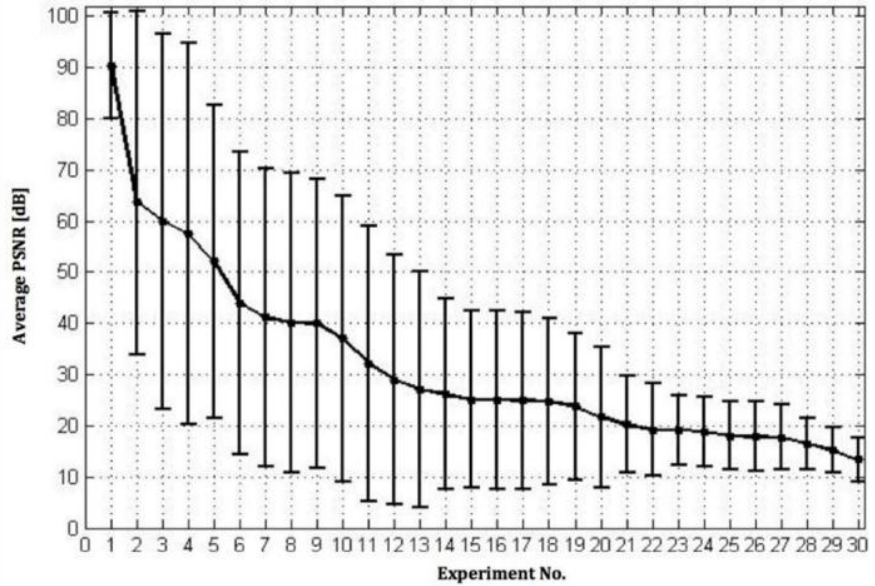

c)

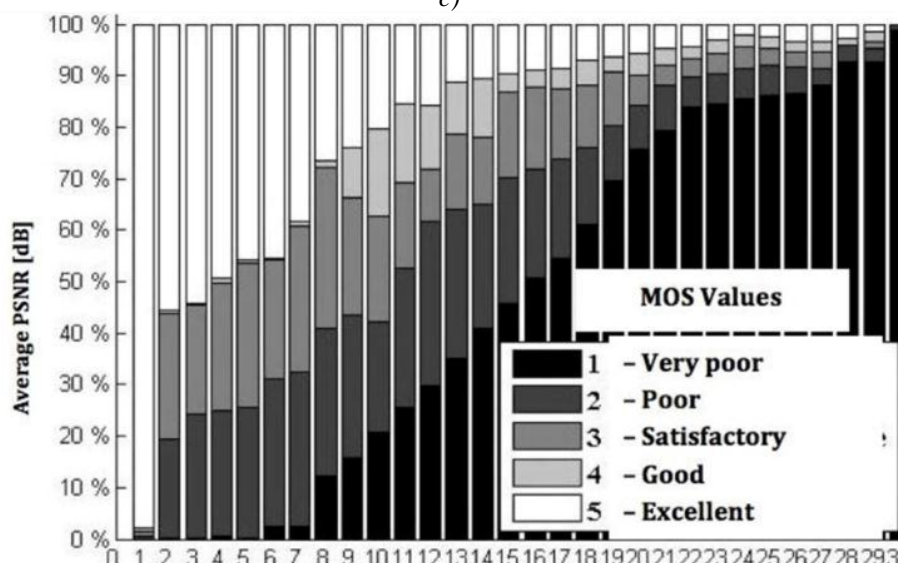

Experiment No.

d)

Figure 2. Values of video sequence quality indicators for different values of wireless channel PER: a) -PSNR value distribution histogram; b) - PSNR value distribution histogram for certain values of PER; c) - Quality deviation from average PSNR value; d) - quality gradation for MOS values. 
when errors in the communication channel are negligible, the PSNR distribution has only one maximum.

Empirical values of BER transitions from an acceptable quality to the poor, according to the relationship between PSNR and MOS [6], are presented in Table II.

However, the AWGN model does not allow for adequately simulation of a fading channel. Typically, errors are often long term, since high probability of bit loss occurs in specific periods of transmission, e.g. during poor propagation. Attenuation of the transmitted signal results in packetizing (grouping) of errors. Another cause of error grouping can be physical defects of, and failures inherent in the information storage system. When using VLC, bit error occurrence results in group errors or packetization of errors.

\section{B. Effect of packet error}

Fig. 3 shows the effect of PER indicator on streaming video quality.

The range of values of PER, within which the resulting quality is maximal (i.e. almost equal to the original) and minimal are indicated. It is shown that with PER $\leq 1 \times 10-4$ error does not affect the resultant video quality and can be easily eliminated with decoders and existing methods of error correction. A further change in the quality has a stepwise nature and decreases with increasing PER.

Empirical values of PER transitions from an acceptable quality to the poor, according to the relationship between PSNR and MOS, are presented in Table III.

TABLE 3. RELATIONSHIP BETWEEN QUALITY INDICATORS AND PER

\begin{tabular}{|c|c|c|c|c|}
\hline $\begin{array}{c}\text { PSNR } \\
{[\mathrm{dB}]}\end{array}$ & $\begin{array}{c}\text { MOS } \\
{[\%]}\end{array}$ & PER & $\begin{array}{c}\text { ITU } \\
\text { Quality Scale }\end{array}$ & $\begin{array}{c}\text { Picture } \\
\text { Degradation }\end{array}$ \\
\hline$>37$ & $81-100$ & $<1 \times 10^{-4}$ & $\begin{array}{c}5 \\
\text { EXCELLENT }\end{array}$ & NOTICEABLE \\
\hline $31-37$ & $61-80$ & $1 \times 10^{-3}-3 \times 10^{-3}$ & $\begin{array}{c}4 \\
\text { GOOD }\end{array}$ & $\begin{array}{c}\text { NOTICEABLE, } \\
\text { BUT NOT } \\
\text { IRRITATING }\end{array}$ \\
\hline $25-31$ & $41-60$ & $3 \times 10^{-3}-1 \times 10^{-2}$ & $\begin{array}{c}3 \\
\text { SATISFACTORY }\end{array}$ & $\begin{array}{l}\text { SLIGHTLY } \\
\text { IRRITATING }\end{array}$ \\
\hline $20-35$ & $21-40$ & $1 \times 10^{-2}-5 \times 10^{-2}$ & $\begin{array}{c}2 \\
\text { POOR }\end{array}$ & IRRITATING \\
\hline$<20$ & $0-20$ & $>5 \times 10^{-2}$ & 1 VERY POOR & VERY IRRITATING \\
\hline
\end{tabular}

Analyzing the results of streaming video over a simulated wireless network with a given probability of packet loss, we safely conclude that:

a) A PER value of $\leq 1 \times 10-4$ in simulation of a wireless network does not affect the video quality. When PER $\leq$ $1 \times 10-3$ impact of errors on video quality is not noticeable and does not irritate during viewing experience. When PER $\geq 0.1$, packet loss in the network has the worst effect on visual quality.

b) Objectively, excellent quality of video transmission over a channel can be guaranteed for all packet error probabilities less than $1 \times 10-3$, good quality is in the

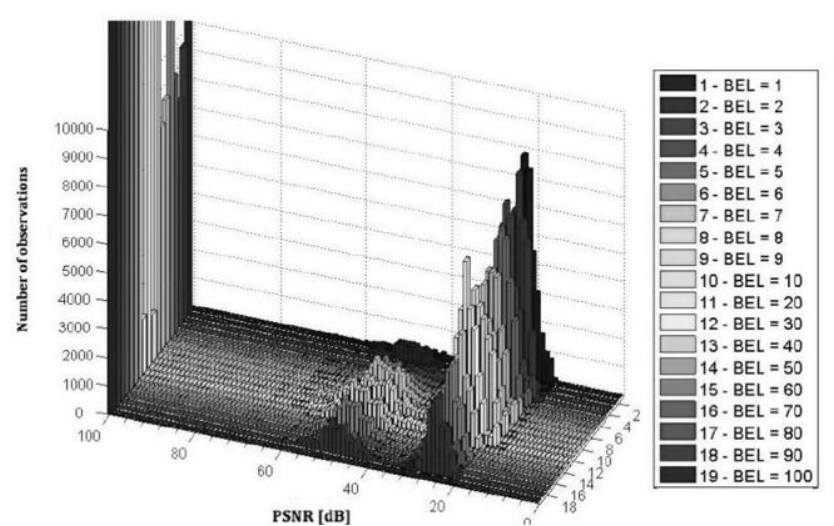

a)

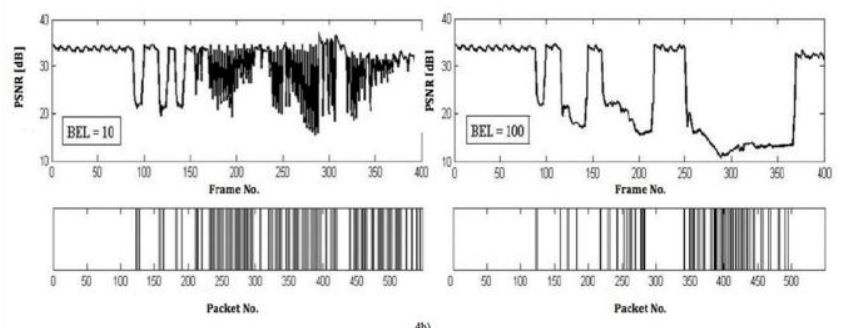

b)
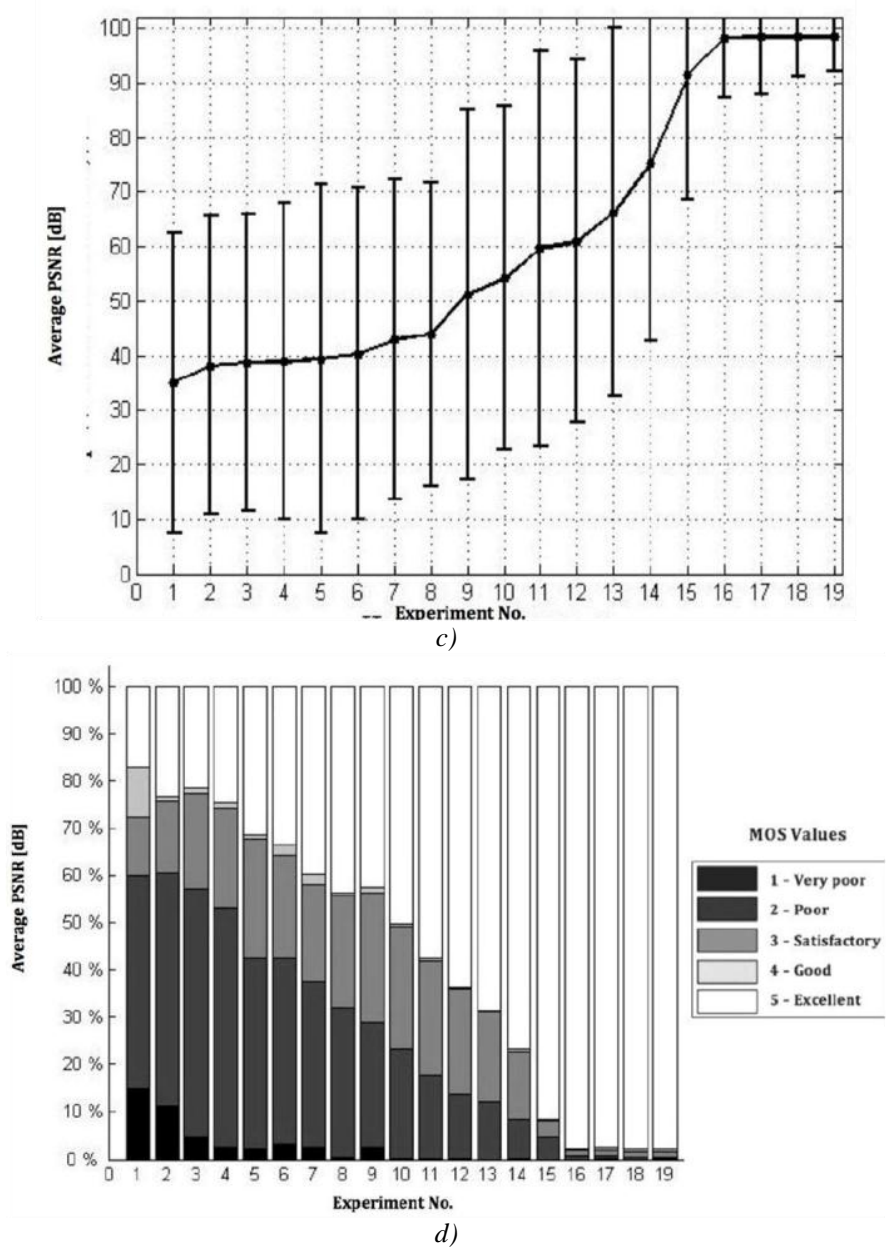

Figure 4. Values of video sequence quality indicators for $P E R=1 \times 10-3$ and varying values of wireless channel BEL: a) -PSNR value distribution histogram; b) - PSNR value and RTP/UDP packet distribution (black spaces correspond to lost packets) for certain values of BEL; c) - Quality deviation from average PSNR value; d) - quality gradation for MOS values. 
range of $1 \times 10-3$ to $3 \times 10-3$, satisfactory quality is in the range of $3 \times 10-3$ to $1 \times 10-2$, poor quality is in the range of $1 \times 10-2$ to $5 \times 10-2$, while very bad quality is for any PER $>5 \times 10-2$.

Histograms of the distribution of values of PSNR when PER $\leq 6 \times 10-4$, in general, have a bimodal shape. One of the peaks characterizes the value of PSNR of video stream distorted due to packet loss. The second maximum characterizes deterioration in the PSNR of dependent frames. As the number of errors increases, one of the peaks increases due to a decrease in the other.

\section{Effect of length of error groups}

To study the effect of the length of error groups on resultant quality, the simulation of a 30-minute video transfer over a wireless network for the values of PER of $1 \times 10^{-3}$ to $5 \times 10^{-2}$ is repeated, since a visual change in video quality is observed at this range. The simulation of groups of error packets during transmission over a wireless channel was done by means of random deletion of packet groups from the receive trace file with a given BEL. For this particular example BEL $=100$ implies that the total random number of consecutively deleted packets does not exceed 100. The total sum of erroneous (deleted) packets in the video sequence for the whole experiment given $\mathrm{PER}=$ const. remained the same, irrespective of the value of BEL. Fig. 4 shows the effect of BEL on the quality of streaming video for $P E R=1 \times 10^{-3}$.

Analyzing the results of streaming video over the simulated wireless network with a given grouping of erroneous packets, we can draw the following conclusions:

v) For PER $\leq 1 \times 10^{-3}$ the effect of single packet errors on quality is insignificant and does not irritate the viewing experience.

vi) Histograms of the distribution of values of PSNR have two maxima. One of the peaks characterizes the value of PSNR of video frames distorted due to the loss of packets. The second maximum characterizes the deterioration of PSNR of dependent frames. With increasing quantities BEL is one of the peaks decreases as the number of dependent frames are also reduced, whereas the second peak remains unchanged. This is explained by the fact that the single scattered throughout the video sequence error number of distorted frames is large due to error propagation to dependent frames. An increase in the BEL value leads to a decrease in one of the maxima, since the number of dependent frames also decreases, while the second maximum remains the same. This is due to the fact that under singular errors spread across the whole video sequence, the number of distorted frames is large because of the distribution of errors on dependent frames.

vii)Increasing the length of the error groups leads to an increase in the average quality of the video sequence.

viii) Effect of error groups on the quality is more powerful because of the local concentration of errors. However, the average quality of the video sequence increases with increase in the length of the grouping for a given value of probability of occurrence of packet errors.

For BEL $\geq 60$ the average quality is almost identical to the original video.

\section{Relationship between PER and BEL}

The average quality of the experimental video sequence for different values of PER and BEL is shown in Fig. 5.

In assessing the impact of erroneous packets received on quality, it is necessary to analyze not only the likelihood of occurrence of errors, but also their structure and length of their grouping. Additionally, the following conclusions can be drawn:

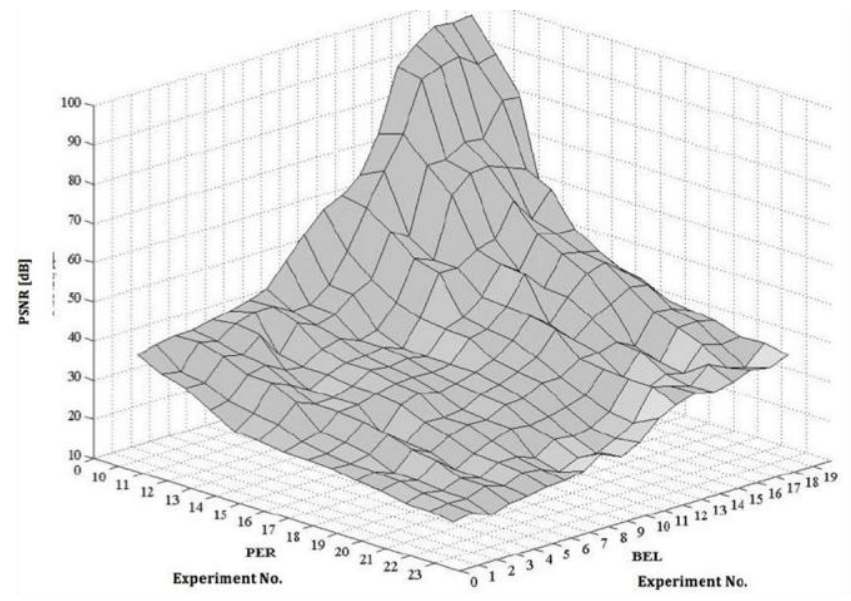

Figure 5. Estimate of video sequence quality indicator for different values of wireless channel PER and BEL.

i) Increasing the length of error groupings leads to an increase in the average quality of the video sequence. This is due to the deterioration of a small section of video, where error groups are concentrated, whereas in the case of single bit errors deterioration in the quality of video may be observed across the whole sequence;

ii) When the length of erroneous packets is BEL $\leq 6$ the change in quality is minor and identical to the influence of single packet errors $(\mathrm{BEL}=1)$;

iii) When BEL $\geq 60$ the average quality is almost identical to the original (PSNR $<90 \mathrm{~dB}$ ). It is logical to assume that the value of BEL in the longer video sequences, with the same average quality may have a higher value;

iv) The highest dynamics of change in PSNR $=60 \mathrm{~dB}$ is observed in two cases: a) for a fixed PER $=1 \times 10^{-3}$ and the variable values of BEL; and $b$ ) at $B E L \geq 80$ and the varying values of the PER. In other cases, the dynamics is not essential and minimal in the absence of clustering of errors $(\mathrm{BEL}=1)$

v) With increasing PER, the effect of BEL on quality decreases due to increase in denseness of single errors.

vi) Analysis of the results of PER and BEL shows that for effective assessment of the impact of transmission errors on resultant quality it is necessary to analyze not only the likelihood of errors, but also their structure and length of their grouping. The most realistic and 
accurate method of modeling statistical errors in communication channels is the use of probability data obtained from real networks.

At BER values $\leq 3 \times 10^{-5}$ bit errors do not affect the quality of the received video and are easily eliminated by well-known methods of error correction implemented in WiMAX. When $\mathrm{BER} \geq 4 \times 10^{-3}$ packet loss in the network reaches its maximum value and leads to an unacceptable quality of the received video. Ensuring objectively excellent quality of video sequence over a channel can be done for probabilities of bit error rate less than $1 \times 10^{-4}$; good quality in the range of $1 \times 10^{-4}-4 \times 10^{-4}$; satisfactory quality in the range of $4 \times 10^{-4}-8 \times 10^{-4}$; poor quality in the range of $8 \times 10^{-4}-1 \times 10^{-3}$ and very bad at BER $\geq 1 \times 10^{-3}$.

The use of H.264/AVC video in wireless access systems with VLC codec of variable length leads to a disruption of the synchronization of decoded video sequences and the occurrence of additional grouping of errors, whose impact on the quality for video decoding is much stronger than that of the bit error, since it leads to loss of large segments of the information. It is shown that the quality of the video affects not only the probability of error, but also the structure and length of errors. Analysis of individual errors showed that at PER $\leq$ $1 \times 10^{-4}$ packet errors do not affect the quality of the received video and are easily eliminated by well-known methods of error correction deployed in wireless networks. When PER $\leq$ $1 \times 10^{-3}$, the effect of errors on quality is not noticeable and does not irritate the viewing experience. For values of PER $\geq 0.1$ packet loss in the network leads to an unacceptable quality of the received video. Ensuring objectively excellent quality of video sequence over a channel can be done for probabilities of bit error rate less than $1 \times 10^{-3}$; good quality in the range of $1 \times 10^{-3}-3 \times 10^{-3}$; satisfactory quality in the range of $3 \times 10^{-3}-$ $1 \times 10^{-2}$; poor quality in the range of $1 \times 10^{-2}-5 \times 10^{-2}$ and very bad at $\mathrm{BER} \geq 5 \times 10^{-2}$.

To assess the impact on quality of video playback under error grouping conditions of error groups BEL, the use of a regular (deterministic) model is proposed. It is shown that the effect of errors on the average quality is stronger due to local concentration of errors. The average quality of the video sequence at the same time increases with increase in the length of the grouping for a given value of probability of occurrence of packet errors. For groupings of length BEL $\geq 60$, average quality is almost identical to that of the source video. With increasing PER, the effect of BEL on quality decreases due to increase in the denseness of single errors. Increase in the BEL leads to an increase in the average quality of the video sequence irrespective of the PER value. The highest dynamics of change in PSNR is observed for fixed PER $=1 \times 10^{-3}$ and the variable values of BEL; at $B E L \geq 80$ as well as for the changing values of the PER. In other cases, the dynamics is not significant and is minimal in the absence of clustering of errors. To assess the quality of video under packetization of errors under real conditions, it is necessary to investigate the actual distribution of packetization of errors in the communication channel.

\section{CONCLUSION}

We have presented in this paper the results of investigating the effect of single bit error and bit packet errors on the quality of H.264/AVC standard bursty video streams in wideband wireless access systems, using a software-hardware composite system that was developed specifically for this purpose. From analyses of simulation results, we safely conclude as discussed in sections VI A through $\mathrm{D}$ for BER, PER, BEL, and relationship between PER and BEL respectively.

\section{REFERENCES}

[1] T. Wiegand, G. J. Sullivan, G. Bjøntegaard, and A. Luthra, "Overview of the H.264/AVC Video Coding Standard", IEEE Transactions on Circuits and Systems for Video Technology, Vol. 13, No. 7, pp. 560576, July 2003.

[2] O. I. Sheluhin, Y. A. Ivanov, "Assessment of the quality of streaming video in telecommunication networks using software-hardware methods," Electro-technical and Information Complexes and Systems, vol. 5, No.4, pp. 48-56, 2009.

[3] Y.A. Ivanov, V.S. Pryanikov, "Imitation Modeling of Wireless Networks using Hardware-Software Complex for the Assessment of Streaming Video Quality," Chuvash University Digest, vol.1, No.1, pp.35-48, 2010.

[4] NS-2 documentation, available at: http://www.isi.edu/nsnam/ns/nsdocumentation.html, accessed 29.06.2010.

[5] G. J. Sullivan, T. Wiegand, "Video Compression-From Concepts to the H.264/AVC Standard", Proceedings of the IEEE, Vol. 93, No. 1, pp.1831, Jan. 2005.

[6] ITU P.800: Methods for subjective determination of transmission quality, available at: http://www.itu.int/rec/T-REC-P.800-199608-I/en.

[7] Atayero A.A., "Estimation of the Quality of Digitally Transmitted Analogue Signals over Corporate VSAT Networks", Ph.D Thesis (unpublished), Moscow, Jan. 2000.

[8] J. Ostermann, et al., "Video coding with H.264/AVC: Tools, Performance, and Complexity", IEEE Circuits and Systems Magazine, pp. $7-28$, Q1. 2004.

[9] J. J. Lemmon, "Wireless link statistical bit error model," NTIA Report. 02-394, U.S. Department of Commerce, June 2002.

\section{AUTHORS PROFILE}

Aderemi A. Atayero graduated from the Moscow Institute of Technology (MIT) with a B.Sc. Degree in Radio Engineering and M.Sc. Degree in Satellite Communication Systems in 1992 and 1994 respectively. He earned a Ph.D in Communications/Signal Processing from Moscow State Technical University of Civil Aviation, Russia in 2000. He is a two-time Head, Department of electrical and Information Engineering, Covenant University, Nigeria. He was the coordinator of the School of Engineering of the same University

Dr. Atayero is a member of a number of professional associations including: the Institute of Electrical and Electronic Engineers, IEEE, the International Association of Engineers, IAENG, among others. He is a registered engineer with the Council for the Regulation of Engineering in Nigeria, COREN, as well as a professional member of the International Who's Who Historical Society (IWWHS). He is widely published in International peer-reviewed scientific journals, proceedings, and edited books. He is on the editorial board of a number of highly reputed technical and scientific publications. He is a recipient of the '2009/10 Ford Foundation Teaching Innovation Award'. His current research interests are in Radio and Telecommunication Systems and Devices; Signal Processing and Converged Multi-service Networks.

Oleg I. Sheluhin was born in Moscow, Russia in 1952. He obtained an M.Sc. Degree in Radio Engineering1974 from the Moscow Institute of Transport Engineers (MITE). He later enrolled at Lomonosov State University (Moscow) and graduated in 1979 with a Second M.Sc. in Mathematics. He received a PhD at MITE in 1979 in Radio Engineering and earned a D.Sc. Degree in Telecommunication Systems and Devices from Kharkov Aviation Institute in 1990. The title of his $\mathrm{PhD}$ thesis was 'Investigation of interfering factors influence on the structure and activity of noise short-range radar'.

He is currently Head, Department of Information Security, Moscow Technical University of Communication and Informatics, Russia. He was the Head, Radio Engineering and Radio Systems Department of Moscow State Technical University of Service (MSTUS). 
Prof. Sheluhin is a member of the International Academy of Sciences of Higher Educational Institutions. He has published over 15 scientific books and textbooks for universities and has more than 250 scientific papers. He is the Chief Editor of the scientific journal Electrical and Informational Complexes and Systems and a member of Editorial Boards of various scientific journals. In 2004 the Russian President awarded him the honorary title 'Honored Scientific Worker of the Russian Federation'.

Yury A. Ivanov was born in Moscow, Russia in 1985. He obtained an M.Sc. degree in Systems, network and devices in telecommunications from Chuvash State University in 2007. He obtained a Ph.D in Telecommunication Networks and Systems in 2011 from Moscow State University of Communication and Informatics. His dissertation topic was "The impact of errors in channels of broadband wireless access systems on the quality of streaming H.264/AVC video". Dr. Ivanov has published over 35 scientific papers and his current research interests include Radio and Telecommunications Systems and Devices: transmission of multimedia data across telecommunication networks, assessment of the quality of video sequences.

Juliet O. Iruemi was born in Kaduna, Nigeria in 1984. She obtained a B.Eng. degree in Information and Communication Technology from Covenant University in 2008. She is currently on her M.Eng. Programme in Information and Communication Technology in Covenant University. Her thesis topic is "Hybrid WLAN Access Point (AP) based on Software Defined Radio (SDR)". Her current research interests include Radio and Telecommunication Systems: Wireless access transmission over broadband network. 